Meta

Journal des tradlucteurs

Translators' Journal

\title{
Problèmes de la traduction juridique au Québec
}

\section{David G. Reed}

Volume 24, numéro 1, mars 1979

La traduction juridique

URI : https://id.erudit.org/iderudit/003933ar

DOI : https://doi.org/10.7202/003933ar

Aller au sommaire du numéro

\section{Éditeur(s)}

Les Presses de l'Université de Montréal

\section{ISSN}

0026-0452 (imprimé)

1492-1421 (numérique)

Découvrir la revue

Citer cet article

Reed, D. G. (1979). Problèmes de la traduction juridique au Québec. Meta, 24(1),

95-102. https://doi.org/10.7202/003933ar d'utilisation que vous pouvez consulter en ligne.

https://apropos.erudit.org/fr/usagers/politique-dutilisation/ 


\title{
Problèmes de la traduction juridique au Québec
}

\begin{abstract}
\& Quiconque voyage à l'étranger sans savoir la langue qu'on y parle s'expose à des bévues en leur attribuant une signification qu'elles n'ont justement pas. \$*
\end{abstract}

Les traductions de textes juridiques pèchent le plus souvent par leur imprécision, alors que dans le domaine du droit, la précision est d'une importance primordiale. Ces imprécisions sont en fait des erreurs de perspective en ce qui concerne le texte original. En général, ces erreurs de perspective sont causées par une lecture hors du sens global ${ }^{2}$ du texte original. Les traducteurs de textes juridiques ne sont que trop rarement des juristes et, quand ils le sont, ils sont par trop éloignés des problèmes linguistiques que pose le texte original. Ces traducteurs ignorent trop souvent les nombreux problèmes d'ordre linguistique que présente le droit du Québec, et sont du même fait ignorants des différents concepts couverts par les mêmes termes. Il est important, à mon avis, de reconsidérer la méthodologie utilisée dans la traduction, qu'elle soit de textes juridiques ou d'autres cuvres non littéraires. Nous retrouvons, en général, deux types d'erreurs commises par la majorité des traducteurs en ce qui concerne la traduction de textes dits techniques, c'est-à-dire de textes n'appartenant pas à la \& Littérature \$: la première est que le texte de départ est souvent traduit en une langue littéraire plutôt que technique, la deuxième est que lorsque le texte d'arrivée $\left(T_{a}\right)$ est rédigé en langue technique, la traduction a été faite d'une façon automatique.

La première erreur est due au fait que les traducteurs possèdent généralement une formation littéraire qui influence leur traduction, de telle sorte que le texte dans la langue d'arrivée est rédigé dans une langue correcte, mais qui ne représente que de très loin les concepts précis et techniques exposés dans le texte de départ. $\mathrm{Si}$, au contraire, les traducteurs sont conscients des difficultés qui existent quand il s'agit de traduire le plus fidèlement possible le texte de départ, ils se retrouvent devant une traduction automatique, c'est-à-dire faite textuellement.

Pour éliminer ces erreurs, une nouvelle manière d'approche de la traduction 《 technique $\gg$ est ici préconisée. Ce que nous appellerons \& traduction technique , est l'opération qui fait correspondre un texte de départ technique à un texte d'arrivée technique. En d'autres termes, le texte de départ présente des difficultés linguistiques qui sont dues non seulement à la structure syntaxique, mais surtout aux lexèmes utilisés, lexèmes appartenant à un domaine spécifique des connais-

* R.-L. Wagner, les Vocabulaires français, Paris, Didier, 1967, t. 1, p. 8.

1. J.-P. Vinay et J. Darbelnet, Stylistique comparée du français et de l'anglais, Montreal, Beauchemin, 1971, p. 161 . 
sances humaines. A l'heure actuelle, cette \& traduction technique » suit plutôt un développement binaire : du texte de départ $\left(T_{d}\right)$ directement au texte d'arrivée $\left(T_{\mathrm{a}}\right): \mathrm{T}_{\text {dep }} \rightarrow \mathrm{T}_{\text {arr }}$. Il est ici suggéré qu'elle devrait être divisée en trois étapes :

a) Tout d'abord la traduction du texte technique de départ $\left(\mathrm{T}_{\mathrm{d}}\right)$ en un texte en prose $\left(\mathrm{t}_{\mathrm{d}}\right)^{2}$, c'est-à-dire paraphrasé et cela, toujours dans la langue de départ.

b) Ensuite, ce texte en prose dans la langue de départ $\left(t_{d}\right)$ sera traduit en un texte en prose dans la langue d'arrivée $\left(t_{2}\right)$.

c) Enfin, en demeurant dans la langue d'arrivée, un transfert de cette version en langue technique $\left(\mathrm{T}_{\mathrm{a}}\right)$.

Ces trois étapes pourraient être schématisées de la manière suivante :

a) $T_{d} \rightarrow t_{d}$

b) $t_{d} \rightarrow t_{a}$

c) $t_{a} \rightarrow T_{a}$

Comme nous l'avons dit plus haut, le traducteur n'est que trop souvent une machine binaire $\left(T_{d} \rightarrow T_{a}\right)$. Il se doit plutôt d'être interprétatif, sa responsabilité étant de * translater » et non pas de traduire, pour en arriver éventuellement à une équation linguistique où le sens des deux membres est quasi identique $\left[' T{ }^{\prime}{ }_{\mathrm{d}} \cong{ }^{\prime} \mathrm{T}{ }_{\mathrm{a}}\right]$. Pour arriver à cette équation, nous suggérons qu'il suive les trois étapes établies ci-dessus.

Pour en arriver à une traduction adéquate, il nous faut être conscients de différents procédés de la pensée. Pour apprendre une langue qui n'est pas la nôtre - en l'occurrence la langue de départ puisque la majorité des traducteurs traduisent dans leur langue maternelle - il s'agit de traverser une barrière culturelle. Il nous faut reconnaître qu'une langue possède de nombreux langages, et que contrôler un de ces langages n'implique absolument pas la maîtrise de tous les autres. Tous ces préceptes mènent éventuellement à la conclusion que pour arriver à une traduction en prose $\left(t_{d}\right)$, l'on doit comprendre dans toute leur ampleur, les détails techniques du texte de départ $\left(\mathrm{T}_{\mathrm{d}}\right)$. Jusqu'à présent, l'on a trop insisté sur la connaissance de la langue d'arrivée littéraire de la part du traducteur, alors qu'il semble préférable sinon indispensable que les connaissances techniques soient égales dans les deux langues. Ce problème culturel constitue la première étape à franchir.

En droit, les nombreuses erreurs de traduction rencontrées sont dues au fait que les traducteurs manquent du temps nécessaire à se pencher sur la traduction adéquate de certains termes et expressions juridiques. Au Canada, comme on le sait, coexistent deux systèmes juridiques : le droit civil du Québec et le « common law > des autres provinces. Ici, le problème de traduction est multiple: les concepts québécois du droit ne peuvent pas facilement se traduire en anglais, du fait qu'il y a interférence entre la langue du * common law $»$ et celle du droit civil. L'inverse est tout aussi vrai en "common law \& comme s'en rendent compte

2. Texte rédigé en langue sémantique, c'est-à-dire utilisant des mots compris intuitivement par toute personne parlant cette langue. 
ceux qui traduisent en français les lois du Nouveau-Brunswick et de l'Ontario. Il s'agira d'unifier les efforts des chercheurs pour en arriver à un langage s civil * et à un langage de « common law bilingues, où chaque lexème représenterait adéquatement un concept dans un de ces langages. Le mot anglais « trust », par exemple, est traduit par le mot français e fiducie \ dans notre Code civil (articles 981b, 981c, 981d, 981f, $981 \mathrm{~g}, . .$.$) . Le terme anglais a les significations suivantes:$

1. a confidence reposed by one person in conveying or bequeathing property to another, that the latter will apply it to a purpose or purposes desired by the former...

2. ... the beneficial interest created by such a transaction... ${ }^{3}$

A right of property, real or personal held by one party for the benefit of another...

A fiduciary relation with respect to property, subjecting person by whom the property is held to equitable duties to deal with the property for the benefit of another person which arises as the result of a manifestation of an intention to create it ${ }^{4}$.

Alors qu'en France le terme fiducie est défini comme suit :

Contrat par lequel l'acquéreur apparent d'un bien s'engage à le restituer à

l'aliénateur quand celui-ci aura rempli les obligations qu'il a envers lui ${ }^{5}$.

Ou encore :

Garantie obtenue par un créancier dans un contrat par lequel il est l'acquéreur apparent d'un bien qui lui est transmis par son débiteur, et qui sera restitué à ce dernier lorsque la dette sera éteinte ${ }^{8}$.

Avec l'emploi du mot * fiducie * au Québec, il ne s'agit bien sû́r pas d'une mauvaise traduction, mais d'un terme qui a changé de signification. Le sens d'un mot français peut s'être modifié selon le pays francophone où ce mot a pu être utilisé. Il est clair que \& trust * et « fiducie » ne sont pas représentatifs d'un même concept juridique. Les deux concepts étant différents, cette mauvaise traduction exemplifie une des difficultés auxquelles on fait face quand cette barrière culturelle mentionnée plus haut se trouve être traversée. L'erreur du traducteur est d'être allé directement au langage "technique * de la langue d'arrivée, sans comprendre dans toute son ampleur la signification des mots « trust \$ et « fiducie 》. S'il avait suivi les trois étapes préconisées, il n'aurait jamais choisi le mot \& fiducie », dont la définition en France a été donnée plus haut, alors qu'au Québec, Bélisle l'explique comme suit :

Disposition par laquelle le fiduciaire est chargé d'administrer un héritage ou des biens à lui confiés jusqu'à une date à laquelle il devra remettre ces biens au véritable légataire ?

Pour résumer, il nous paraît utile de citer le professeur $D$. Waters :

When the civilian sees common lawyers attempting to fit the interest of the trust beneficiary into these civil law inspired categories of in rem and in

3. John B. Saunders, Mozley and Whiteley's Law Dictionary, London, Butterworths, 1970.

4. Henry Campbell Black, Black's Law Dictionary, St. Paul, West Publishing Company,

1968.

5. Henri Capitant, Vocabulaire juridique, Paris, P.U.F., 1951

6. R. Guillien et J. Vincent, Lexique de termes juridiques, Paris, Dalloz, 1974.

7. L.-A. Bélisle, Dictionnaire général de la langue française au Canada, Bélisle, 1974. 
personam rights, and disagreeing among themselves as to which category, if either, it properly falls into, he fairly concludes that neither for theoretical nor for practical purposes can the trust beneficiary's interest be described as only an in personam right, and received into the civil law system the trust might produce an open-ended list of in rem rights. Seen from any angle, he may conclude, it is going to be a cuckoo in the nest ${ }^{8}$.

Ou encore :

Unlike the common law system the civil law does possess a doctrine of absolute ownership, and because of this there is no way in which a civil law system can exactly reproduce the common law trust ?

Un autre exemple serait le terme anglais de « ownership ». Il est traduit par * propriété > dans le Code civil (articles 405, 406, 408...). Il découle de notre étude sur * trust \$ que les termes « ownership 》 et "propriété » en «common law » et en droit civil couvrent des concepts différents. Le concept français de * propriété \$ est indivis, c'est-à-dire non divisible, alors qu'en « common law », \& ownership 》 peut l'être grâce à la notion d'« estates »:

In modern civil law, a principle derived from Roman sources, it is axiomatic that ownership of property involves the owner in having all the rights associated with ownership, namely disposition, management, and enjoyment. If any one of those rights is severed, by definition ownership does not exist... The civil law has no doctrine of estates, and it has therefore no conception of dividing up the rights associated with ownership among two or more persons ${ }^{10}$.

Un dernier exemple serait la traduction de l'anglais \& real rights \$ par \& droit réel \ dans notre Code (articles 809, 1208, 2088, 1353a, 804, 805, 2015, 2016, $2017,2026,2034,2082,2083,2092,941 \ldots)$. En anglais, « real $\gg$ évoque exclusivement ce qui appartient à la terre. Le Code civil a adopté cette signification quand il parle de " real property » ou propriété foncière (article 1939), ou encore dans l'article 499 , où * héritage » est traduit par \& real estate ». Comme le suggère Black :

In Civil law, real is defined as relating to a thing (whether moveable or immoveable) as distinguished from a person ${ }^{11}$.

La défiinition de « real » en « common law », toujours d'après Black est :

Relating to land, as distinguished from personal property. This term is applied to lands, tenements, and hereditaments ${ }^{12}$.

Il existe deux raisons pour lesquelles les termes « real estate \$ et « real property \$ ne devraient pas être utilisés en droit civil. Tout d'abord, pour la raison mentionnée ci-dessus, « real » est à éviter, et ensuite « estate » ne devrait pas être utilisé puisque le mot représente un concept inconnu aux juristes civilistes (par exemple les termes « fee simple» et « fee tail » qui appartiennent au concept

8. D.M.W. Waters, Law of Trusts in Canada, Toronto, The Carswell Company Limited, 1974 , p. 934.

9. Ibid., p. 15.

10. Ibid., p. 15-16.

11. Henry Campbell Black, Black's Law Dictionary, déjà cité.

12. Ibid. 
d'\& estates » sont absolument inconnus ici, alors qu'ils sont utilisés dans le * common law »). Il nous suffira de lire la définition de droit réel telle qu'elle nous est fournie par les professeurs Kouri et Archambault pour voir que l'expression peut s'appliquer à un droit sur meuble ainsi que sur immeuble :

Même si les accessoires d'une créance comme le droit de gage ou le droit d'hypothèque sont des droits réels, le principal, c'est-à-dire la créance même, reste toujours un droit personnel.

Mazeaud nous donne le principe suivant : Le droit de gage ou le droit d'hypothèque confère aux créanciers-gagistes ou aux créanciers-hypothécaires, un droit direct sur le meuble donné en gage ou sur l'immeuble hypothéqué. Ce droit réel est dit accessoire par ce qu'il est l'accessoire d'une créance (Mazeaud, H.L., Leçon de droit civil, $4^{\mathrm{e}}$ éd., Paris, Editions Montchrestien, 1968 , t. 1, vol. 1 , no 163 , p. 192) ${ }^{13}$.

Nous rencontrons un problème similaire avec * real action». Toujours d'après Black :

At common law, one brought for the specific recovery of lands, tenements, or hereditaments... Among the civilians, real actions, otherwise called * vindications \$, were those in which a man demanded something that was his own. They were founded on dominion, or jus in $\mathrm{re}^{14}$.

Il nous est facile de reconnaître, d'après ces exemples multiples, les problèmes linguistiques et juridiques auxquels font face les traducteurs juridiques. Il est clair que nous avons un besoin impérieux de préciser les concepts, afin justement de représenter par un lexème, et un seul, un concept de droit spécifique.

La deuxième étape est celle de la traduction littérale. Il est évident que ce problème est présent dans la quasi-totalité des textes traduits dans n'importe quelle langue, et c'est un des obstacles les plus difficiles à surmonter pour un traducteur. Celui-ci doit pouvoir s'éloigner suffisamment de la langue de départ pour pouvoir se pencher sur l'expression du signifié dans la langue d'arrivée. Très souvent, il semble faire preuve d'un effort conscient de ne pas justement commettre cette erreur. Malheureusement, cet effort provoque une langue presque incompréhensible si l'on ne possède pas la version dans l'autre langue. Un exemple de ce problème serait l'article $\mathbf{5 5 0}$ de notre Code. Il se lit comme suit dans les deux langues :

Le titre constitutif de la servitude ne peut être remplacé que par un acte recognitif émanant du propriétaire du fonds asservi.

The want of a title creating the servitude can only be supplied by an act of recognition proceeding from the proprietor of the land subject thereto.

Cet article nous vient de l'article 695 du.Code Napoléon qui se lit comme suit :

Le titre constitutif de la servitude à l'égard de celles qui ne peuvent s'acquérir par la prescription, ne peut être remplacé que par un titre recognitif de la servitude, et émane du propriétaire du fonds asservi ${ }^{25}$.

13. Robert Kouri et Denis Archambault, Droit civil. Le droit des biens et la preseription, Sherbrooke, Université de Sherbrooke, Faculté de droit, année universitaire 1974-1975, p. 7.

14. Henry Campbell Black, Black's Law Dictionary, déjà cité

15. J.J. Beauchamp, The Civil Code of the Province of Quebec Annotated, Montreal, Theoret, 1904, vol. 1, p. 458. 
A la lecture de cet article; de sa traduction en anglais et de l'article qui a donné naissance à celui que nous trouvons dans notre Code, la différence entre les versions française et anglaise est évidente. Pour parler plus spécifiquement, l'expression française « titre » contraste avec l'anglais \& want of title». Tout d'abord, notre article 550 nous vient du Code Napoléon. Historiquement, « want of title » décrit la situation où le titre de propriété ne peut être trouvé.: Nous pourrions donc être d'accord avec la version anglaise puisqu'elle coïncide avec la doctrine ${ }^{16}$. Cependant, un linguiste est troublé par cette traduction, puisque l'article n'a pas la même signification dans les deux langues. Cette signification différente pourrait être due à l'interférence du « common law » en droit civil. L'article qui précède directement celui qui nous intéresse stipule que \& nulle servitude ne peut s'établir sans titre... ${ }^{1 \tau}$ Cependant, want of title 》 pourrait nous venir du \& common law ». D'après Black, \& title > est défini comme « the union of all elements which constitute ownership ${ }^{18}$ ». Le même dictionnaire définit « servitude » comme suit :

When the freedom of ownership in land is fettered or restricted, by reason of some person, other than the owner thereof, having some right therein, the land is said to serve s such person. The restricted condition of the ownership or the right which forms the subject-matter of the restriction is termed a \& servitude ${ }^{19}$. *

Nous voyons qu'en \& common law \& une \& servitude * est bien un droit, mais elle n'accorde aucun titre, d'où l'anglais «want of title » qui pourrait donc être le résultat de la terminologie du « common law \& entrant en conflit avec le droit civil. Une autre possibilité serait l'hypothèse que la version anglaise n'est qu'un éclaircissement de l'article 550 de notre Code qui ne fait que reproduire, en omettant une phrase qui nous permettrait d'y voir un peu plus clairement, l'article 695 du Code Napoléon. De toute façon, l'étude de la langue de cet article a révélé un manque de précision dans sa rédaction qui a donné naissance à notre article 550 et subséquemment à la traduction erronée en anglais.

Ce problème de correspondance d'un concept « civiliste 》 avec un concept du * common law $\gg$ relève de la lexicologie. Il est évident que l'absence de matériel de référence en ce qui concerne le droit civil québécois constitue la difficulté fondamentale à laquelle font face les traducteurs de textes juridiques. Pour pallier cette insuffisance, un lexique des termes juridiques utilisés au Québec a certainement sa place.

Tout d'abord, il fut décidé par le Centre de droit comparé de l'Université McGill de rédiger un lexique se basant seulement sur la terminologie juridique telle qu'elle est utilisée dans notre Code civil québécois. Ceci ne fut certainement pas chose aisée à faire. Certains, sinon tous les problèmes auxquels font face les terminologues du Québec sont présents dans le lexique. Ce dernier débuta comme une préparation à un cours sur la terminologie juridique française utilisée au Québec, cours donné à la Faculté de droit de l'Université McGill depuis 1975. Vu que les étudiants s'efforçaient d'apprendre la terminologie juridique du Québec par l'entre-

16. P.B. Mignault, le Droit civil canadien, Montréal, 1895-1916, t. 3, p. 148.

17. Code civil de la province du Québec, article 549 .

18. Henry Campbeli Black, Black's Law Dictionary, déjà cité.

19. Ibid. 
mise de textes venant de France, entre autres, ils éprouvaient beaucoup de difficultés à assimiler ce langage continental, à bien des égards différent de celui qui est utilisé dans les textes juridiques et tribunaux québécois. Après quelques conversations avec des juristes de la Faculté de droit, le Centre de droit comparé nous octroya une subvention nous permettant d'inventorier les termes et expressions pouvant faire partie d'un lexique juridique bilingue éventuel. Notre tâche fut donc de créer un instrument de recherche éventuel ayant également un fonds normalisateur et non seulement d'inventorier les termes juridiques présents dans notre Code, mais encore de suggérer, le cas échéant, la traduction qui représenterait le plus précisément possible le concept du signifié « civiliste». Le choix de ces entrées présenta des difficultés. Bien que le sujet transcendant fût le droit, il se trouva bien sûr des lexèmes tels que \&nonobstant 》, \& à l'encontre de », utilisés presque exclusivement dans le langage juridique, et qui trouvèrent leur place automatiquement à côté de termes plus évidemment juridiques comme "biens *, 4 divorce \$, etc. Le dépouillement souleva le problème de faire la discrimination entre les lexèmes ayant leur place dans un lexique général de la langue française ou anglaise. Le choix fut fait de n'inclure que des termes exclusivement juridiques (et ce, bien sûr, après des consultations éventuelles avec des juristes), qui soient des expressions courantes représentant des concepts juridiques enseignés dans les cours de droit. Nous avons également choisi d'écarter ceux qui, bien qu'utilisés en droit, trouveraient leur place plus naturellement dans un dictionnaire non spécialisé. Les quatre problèmes auxquels nous dûmes faire face furent tout d'abord le choix des lexèmes suivant deux critères : leur place au sein d'un lexique juridique et la qualité du français utilisé dans le Code. Ensuite, il fallut limiter le nombre d'entrées pour que le lexique soit facilement utilisable par les avocats. Le troisième problème consista à vérifier la correspondance des traductions (et nous avons montré ci-dessus l'énorme difficulté que cela représente). Enfin, il nous fallut nous pencher sur la question de la correction des fautes d'usage, de langue, présentes dans un document aussi public que notre Code civil.

Le gouvernement du Québec a lancé une campagne de francisation voici quelque temps. Pour poursuivre dans ce même but, quelle langue faut-il choisir ? Celle d'emploi au Québec, avec certaines expressions comme "actionner», par exemple, utilisée par les avocats au lieu \& d'intenter une action 》 ou «agir » tout simplement? L'influence anglophone est incontestable, et le problème des deux droits canadiens vient s'y ajouter : des concepts du.droit civil sont représentés en anglais par des termes venant du " common law \$, comme * trust $\$$ traduit par - fiducie $\gg$; à cela s'ajoute le problème de termes « consacrés » par le Code, mais qui sont des traductions de termes anglais ou même de termes désuets qui ne sont plus utilisés " papier-nouvelles » pour « newspaper» $(1571 \mathrm{aCC})$.

Si le but de francisation doit être atteint, il s'agira bien sâr de fixer, du moins pour le moment, cette langue française qui semble nous échapper et qu'il est très difficile de cerner. Il faudra éventuellement qu'un groupe de linguistes et de spécialistes - des juristes praticiens et théoriciens en l'occurrence - se rencontrent et décident de la forme que prendra la langue juridique française du Québec. Il est toujours possible de la laisser voguer à la dérive comme ce fut 
le cas jusqu'à présent. Si le français doit survivre au Québec, il faut admettre son identité, son entité, son «unicité » au sein du monde anglophone. Nous nous devons de définir exactement les problèmes auxquels nous faisons face dans la traduction du langage juridique du Québec pour pouvoir éventuellement limiter, sinon éliminer, les erreurs purement linguistiques ou qui s'attachent plutôt à des questions de droit. Le dépouillement nous a montré que pour arriver à fixer la langue québécoise, il nous faut inventorier les difficultés de traduction et les contresens du Code civil pour ensuite les classifier et ainsi mettre à nu les problèmes présents dans les différents domaines de la traduction juridique.

Nous espérons que la prochaine publication de notre lexique permettra aux traducteurs de textes juridiques d'appliquer plus facilement la méthodologie que nous avons préconisée plus haut. Comme il contiendra une partie normalisatrice, c'est-à-dire que nous nous permettrons de suggérer certaines traductions qui nous semblent représenter plus précisément le concept original, il devrait faciliter la tâche des traducteurs juridiques. Le centre de droit comparé de l'Université McGill vient d'obtenir une subvention de l'Office de la langue française pour rédiger un dictionnaire avec définitions explicatives qui, jumelé avec le lexique dont nous venons de parler, devrait permettre de mener à bien le processus de traduction suggéré.

DAVID G. ReED 